\title{
ANÁLISE DO FLUXO DE VALOR SOB UMA PERSPECTIVA ESTOCÁSTICA
}

\section{VALUE STREAM ANALYSIS FROM A STOCHASTIC PERSPECTIVE}

\author{
Leonardo Bitencourt* E-mail: leonardobittsouza@gmail.com \\ Guilherme Luz Tortorella* E-mail: gtortorella@bol.com.br \\ Daniel Nascimento** E-mail: nascimentodaniel63@gmail.com \\ * Universidade Federal de Santa Catarina (UFSC), Florianópolis, SC \\ Universidade Federal Fluminense (UFF), Niterói, RJ
}

\begin{abstract}
Resumo: A aplicação da produção enxuta $(\mathrm{PE})$ surge como uma resposta à busca por aumento da competitividade em um mercado globalizado. Dentre as práticas utilizadas na aplicação da PE, o mapeamento do fluxo de valor (MFV) serve como meio para identificar desperdícios e direcionar a aplicação das demais práticas, sendo, consequentemente, uma das mais adotadas. O MFV permite mapear fluxos de materiais e informações de controle da produção. No entanto, o MFV possui uma abordagem determinística, o que limita o poder de modelagem e dificulta o mapeamento de modelos dinâmicos. Nesse sentido, este trabalho objetiva a proposição de um método de identificação de oportunidades de melhorias que combine o mapeamento de fluxo de valor e simulação de Monte Carlo, de modo a levar em consideração as incertezas do fluxo de valor na sua análise de lead time. Os resultados obtidos mostram que essa nova abordagem permite identificar oportunidades de melhoria que não seriam contempladas em uma aplicação tradicional do MFV, possibilitando atuar não somente em valores nominais dos processos, mas também em sua variação.
\end{abstract}

Palavras-chave: Sistema de Produção Enxuta. Mapeamento de Fluxo de Valor. Simulação de Monte Carlo.

Abstract: Lean Production (LP) emerges as a response to the increase in competitiveness in a globalized market. Among the practices utilized in LP, value stream mapping (VSM) is one of the most adopted, as it identifies wastes and direct the application of other LP practices. VSM maps material flows and production control information. However, VSM has a deterministic approach, which limits the power of modeling and makes it difficult to map dynamic models. In this sense, this work has the goal of proposing a method for the identification of opportunities for improvements that combine VSM and Monte Carlo simulation, taking into account the uncertainties of the value flow. The results show that this new approach allows the identification of improvement opportunities that are not contemplated in a traditional application of the VSM.

Keywords: Lean production. Value stream mapping. Monte Carlo simulation.

\section{INTRODUÇÃO}

O termo produção enxuta (PE), definido na década de 90 por pesquisadores do Massachusetts Institute of Technology, refere-se à estratégia de aumentar o valor oferecido ao cliente através do uso mais eficiente dos recursos da organização (GREEF et al. 2012). De acordo com Womack e Jones (2004), o pensamento enxuto 
pode ser definido através de cinco princípios: especificação do valor do ponto de vista do cliente; identificação do fluxo de valor; fazer o valor fluir; fazer com que a produção seja puxada pela demanda; buscar a perfeição. Muitas empresas vêm buscando a implementação da PE como uma resposta da organização para a crescente competitividade em uma mercado global (ABDULMALEK; RAJGOPAL, 2007); WOEHRLE; ABOU-SHADY, 2010). Os resultados obtidos a partir da implementação da PE vêm demonstrando consistentemente as vantagens em sua adoção, com melhorias na produtividade, redução do lead time, aumento da satisfação dos clientes e redução da rotatividade dos colaboradores (JIMMERSON et al., 2005).

Dentre as técnicas existentes para a implementação enxuta, o mapeamento de fluxo de valor (MFV) visa identificar todas as atividades envolvidas (de valor agregado ou não) ao longo dos fluxos de material e informação de uma família de produtos específica (WOMACK; JONES, 2004). Nesse sentido, o MFV identifica as atividades que adicionam ou não valor no processo através do estudo dos recursos chave envolvidos no processo, tais como pessoas, materiais e informações (JIMMERSON; WEBER; SOBEK, 2005). De acordo com Duggan (2002), o MFV fornece uma estrutura que garante a melhoria contínua. Através da aplicação do MFV, é possível analisar o fluxo de valor e garantir que as ações implementadas serão realizadas nos pontos onde há maiores oportunidades e trazem benefícios reais ao negócio. Exemplos de aplicação do MFV podem ser achados no setor hospitalar (TORTORELLA et al., 2015; DICKSON et al., 2009; JIMMERSON et al., 2005), em indústrias de processamento contínuo (ABDULMALEK; RAJGOPAL, 2007), desenvolvimento de produto (TYAGI et al., 2015) e na área administrativa (TEGNER et al., 2016).

Contudo, cabe destacar algumas limitações envolvidas quanto ao uso desta técnica. Estudos que abordam o MFV costumam basear suas informações e inferências de melhorias a partir de uma perspectiva determinística, tais como Tortorella et al. (2015), Tyagi et al. (2015) e Dickson et al. (2009). Em outras palavras, ao longo do fluxo de valor há várias fontes de incerteza, tais como estoques, tempos de processamento e setup, entre outras, as quais adicionam variabilidade aos processos de manufatura (STANDRIDGE; MARVEL, 2006), dificultando o gerenciamento da produção. Dentre as consequências de um cenário produtivo com alta variabilidade, o aumento dos níveis de estoque surge como uma contramedida para mitigar os efeitos dessa variabilidade. A adição de estoques aumenta o lead time 
e custos dos processos, além de mascarar o problema principal que são as fontes de incerteza (MAPES et al., 2000).

Além disso, o direcionamento de melhorias a partir de cenários determinísticos pode levar a ganhos pontuais sem de fato agir em questões fundamentais para o desempenho do fluxo de valor (STANDRIDGE; MARVEL, 2006). Métodos quantitativos que consideram a estocacidade dos processos permitem uma visão mais realista quanto às variabilidades existentes, identificando meios para mitigar tais fontes de incerteza. Assim, de forma a complementar o MFV, estudos anteriores o têm integrado a outras técnicas. Braglia et al. (2009) utilizam o MFV e através de lógica fuzzy e de métodos probabilísticos tratam as variabilidades contidas nos processos. Woehrle e Abou-shady (2010) apresentam a utilização do MFV e do Lean Accounting (Contabilidade Enxuta) em conjunto com simulação, de forma a tratar a incerteza e os aspectos financeiros da implementação da PE. A incorporação dessa variabilidade ao MFV permite que as incertezas sejam tratadas e a reduzidas, acarretando em menores níveis de estoque, lead time e custos operacionais (FLYNN et al. 1995).

Nessas situação de maior variabilidade, a solução normalmente empregada é a de utilização dos menores e maiores valores para que sejam encontrados os cenários de pior e melhor caso na estimativa do lead time (BRAGLIA et al. 2009). As práticas da PE visam a remoção da variabilidade e o consequente ajuste dos níveis de estoque, conforme as observações feitas no decorrer do tempo. Nesse sentido, as abordagens comumente encontradas ajustam as características dos processos produtivos com base em tentativa e erro (STANDRIDGE; MARVEL, 2006), o que costuma implicar em um maior tempo para efetuar melhorias realmente significativas para o sistema produtivo. Nesse sentido, o uso de simulação em conjunto com o MFV serve como uma solução para o caráter estático do mesmo (LIAN; VAN LANDEGHEM, 2007).

Assim, este artigo visa propor um método de identificação de oportunidades de melhorias que combine o mapeamento de fluxo de valor e simulação de Monte Carlo. A contribuição deste estudo compreende a identificação das principais fontes de incerteza existentes no fluxo de valor e a definição do impacto de sua variabilidade no lead time do fluxo através da consideração de suas distribuições de probabilidade. Segundo Braglia et al. (2009), um dos cenários onde é necessário incorporar técnicas ao MFV é quando os processos em análise possuem alta variabilidade, já que o não 
tratamento das variabilidades contidas nos processos constitui-se como uma das principais limitações apresentadas pelo MFV. Tratar as variabilidades é importante já que esta é inerente aos tempos dos processos da maior parte das indústrias (MAPES et al. 2000). Assim, o método proposto é ilustrado a partir de uma aplicação prática em um fluxo de suprimentos em um hospital-escola público brasileiro.

\section{MAPEAMENTO DE FLUXO DE VALOR}

De acordo com Rother e Shook (1999), o fluxo de valor envolve qualquer ação necessária para transformar a matéria-prima em produto acabado. O MFV é utilizado para o mapeamento de um processo produtivo ou uma cadeia de suprimentos (SETH; GUPTA, 2012), sendo capaz de mapear não apenas o fluxo de materiais, mas também o fluxo de informações que controla a produção e a movimentação destes (BRAGLIA et al., 2006). O MFV é desenvolvido de forma a apresentar claramente o processo em estudo e suas principais características, evidenciando os problemas existentes (TYAGl et al., 2015) e capturando todas as atividades envolvidas (SETH; GUPTA, 2012). Seu objetivo é, através do estudo desses fluxos, identificar oportunidades para reduzir os desperdícios de forma a impactar mais significativamente o bottom line da empresa (FORNO et al., 2014).

A aplicação do MFV permite a identificação de oportunidades sistêmicas, garantindo que os esforços na implementação de melhorias tragam resultados para o fluxo como um todo, e não apenas pontualmente. Devido a esse seu caráter holístico, o MFV é uma das práticas mais utilizada da PE (MARODIN; SAURIN, 2013) e serve como base para a implementação de outras práticas da PE (SETH; GUPTA, 2012), sendo também utilizado como base em processos de tomada de decisão (HINES et al., 1998).

Rother e Shook (1999) apresentam as etapas necessárias para a aplicação do MFV: (I) definir a família de produtos a ser mapeada; (ii) percorrer o chão de fábrica de modo a elaborar o mapa de estado atual; (iii) analisar as informações coletadas e o estado atual de forma a elaborar o mapa de estado futuro; (iv) desenvolver um plano de ação de forma a detalhar os passos a serem efetuados para que o estado futuro seja atingido. Uma vez que o estado futuro é alcançado, o ciclo recomeça, sendo este o novo estado atual do fluxo. 
De acordo com (SINGH et al., 2011), uma importante parte do MFV é o registro das relações entre os processos de manufatura e seus mecanismos de controle, tais como informações dos produtos e planejamento da produção. Diferentemente da maioria dos processos de mapeamento, que documentam apenas o fluxo de materiais, o MFV registra informações chaves para o processo, tais como materiais armazenados, pontos de programação e fluxo de informações. Além dessas características, também é possível visualizar no MFV aspectos importantes do processo tais como os tempos de ciclo e de processamento, bem como os materiais envolvidos. Sendo assim, o MFV complementa abordagens de mapeamento convencionais (TYAGI et al., 2015; SETH; GUPTA, 2012).

Diversos exemplos de aplicação de MFV são encontrados em empresas manufatureiras (ABDULMALEK; RAJGOPAL, 2007; LIAN; VAN LANDEGHEM, 2007; BRAGLIA et al., 2009; SETH; GUPTA, 2012); serviços de saúde (DICKSON et al., 2009; TORTORELLA et al., 2015); cadeia de suprimentos (SETH et al., 2008; WEE; WU, 2009), entre outros. Particularmente, Tyagi et al. (2015) utilizam o MFV em um processo de desenvolvimento de produto, associação muito pouco explorada na literatura. De acordo com esses autores, o time-to-market (tempo para disponibilização ao mercado) é ponto chave para o sucesso de um novo produto e o MFV surge como uma alternativa para ajudar na diminuição desse tempo. No entanto, apesar dos diversos benefícios evidenciados, o MFV apresenta algumas limitações e desafios em sua implementação.

De acordo com Hines et al. (1998), uma das dificuldades enfrentadas na aplicação do MFV é a limitada disseminação e compreensão da PE e seus princípios através da empresa como um todo. Dessa forma, a aplicação do MFV sem conciliá-lo com a estratégia da empresa leva a ganhos operacionais, porém sem retornos significativos. Braglia et al. (2006) comentam haver dificuldade na aplicação do MFV em indústrias onde há múltiplos fluxos de valor que se sobrepõem, compartilhando recursos. Forno et al. (2014) afirmam que quando há múltiplos produtos, um dos desafios na sua implementação é a definição do produto a ser escolhido. Woehrle e Abou-Shady (2010) argumentam que um dos primeiros desperdícios a ser identificado e eliminado na aplicação do MFV é o excesso de estoques e destacam que a simples redução destes estoques sem de fato a implementação de melhorias consistentes no 
fluxo de valor pode afetar negativamente os resultados operacionais da empresa, desmotivando a adoção da PE.

O MFV também apresenta como característica a dificuldade em prever quais serão os benefícios ao implementar o que é definido no mapa futuro (LIAN; VAN LANDEGHEM, 2007). A adoção da PE em uma empresa tradicional de manufatura traz uma série de possíveis mudanças, tais como o gerenciamento dos funcionários, layout da planta, fluxo de informações e de bens, e no planejamento e controle da produção (DETTY; YINGLING, 2000). Esses fatores dificultam a aceitação das melhorias e é necessário desenvolver uma alternativa que apresente bases quantitativas para tornar o processo de decisão mais confiável (ABDULMALEK; RAJGOPAL, 2007).

Segundo Mcdonald e Aken (2002), na aplicação do MFV há também a dificuldade na previsão de fluxos e níveis de estoque devido ao seu caráter determinístico, que limita o poder de modelagem e dificulta o mapeamento de sistemas com comportamento dinâmico, que contenham alto grau de variabilidade (LIAN; VAN LANDEGHEM, 2007). De acordo com Braglia et al. (2009), a variabilidade é um fator que impacta diretamente o tempo de ciclo, sendo difícil localizar sua origem e mais difícil ainda reduzi-la. A variabilidade é propagada e ampliada ao longo do processo, impactando diretamente a performance do mesmo. Segundo Forno et al. (2014), a aplicação do MFV em um processo com alta variabilidade dificulta a sua melhoria, já que o mapeamento não representa o comportamento real desse processo.

Nesse sentido, técnicas complementares foram desenvolvidas para ampliar o escopo de aplicação do MFV. Tais técnicas podem ser separadas em dois grupos: simulação e mapeamento detalhado. O primeiro grupo (simulação) possui uma interface voltada a criação de modelos e permite que aspectos dinâmicos dos sistemas produtivos sejam analisados. Através da simulação é possível desenvolver modelos futuros e compará-los ao modelo atual. A combinação do poder computacional e do poder visual do MFV possibilita que a adoção da PE seja mais rápida e a resistência mais baixa (LIAN; VAN LANDEGHEM, 2007; WOEHRLE; ABOU-SHADY, 2010). Abdulmalek e Rajgopal (2007), por exemplo, adaptam os princípios da PE para serem aplicados em uma indústria de processamento contínuo, utilizando a simulação para comparar os estados atual e futuro previsto no MFV. Lian 
e Van Landeghem (2007) desenvolvem uma estrutura modular para a simulação do MFV, podendo ser alimentada através do próprio sistema de gestão da empresa.

O segundo grupo de técnicas (mapeamento detalhado) compreende novos métodos de mapeamento que complementam o MFV. Braglia et al. (2006) propõem uma nova abordagem para a aplicação do MFV em indústrias com produções de alta variedade, através de um processo iterativo e do uso da lista de materiais temporizada. Através dessa lista identifica-se o caminho crítico de produção, o qual é então melhorado e origina um novo caminho crítico. Hines e Rich (1997) sugerem sete técnicas para lidar com a análise de fluxo de valor, sendo que a maioria destas são adaptações de técnicas já presentes em outras áreas, tais como logística, dinâmica de sistemas e gerenciamento de operações. Braglia et al. (2009), por sua vez, propõem dois métodos para a incorporação da variabilidade ao MFV. O primeiro através da obtenção de distribuições beta dos tempos do processo, utilizando como dados de entrada os valores mínimos, médios e máximos presentes nos processos. O segundo método é através da lógica fuzzy, com distribuições triangulares de probabilidade, mais simples que o primeiro método.

\section{MÉTODO PROPOSTO}

O método proposto neste trabalho é compreendido por 6 etapas: (I) identificar a família de produtos, (ii) mapear o fluxo de valor atual desta família, (iii) levantar e mensurar as fontes de incerteza críticas ao fluxo de valor atual, (iv) analisar criticamente o fluxo como um todo e elencar oportunidades de melhoria, $(v)$ desenhar mapa do estado futuro almejado para a família, e ( $v i)$ consolidar ações prioritárias para melhoria do fluxo.

A etapa ( $($ ) consiste na identificação da família de produtos a ser mapeada. Para tanto, é necessário definir os produtos que compõem cada família e seus respectivos processos. Nesse sentido, Duggan (2002) sugere a utilização de uma matriz de produtos e processos que identifique quais produtos seguem os mesmos processos. Consideram-se produtos de uma mesma família aqueles que apresentarem uma familiaridade de processos mínima de $80 \%$. Assim, dentre as famílias identificadas, definem-se critérios para priorizar seus mapeamentos e análise, os quais podem ser 
participação no faturamento, volume de produção, entre outros (TORTORELLA et al., 2016).

Na etapa (ii) é feito o mapeamento do estado atual da família escolhida na etapa anterior. A simbologia e metodologia a serem utilizadas nesse passo são propostas por Rother e Shook (1999). O mapeamento do estado atual permite a visualização das perdas ao longo do processo e a identificação de oportunidades de melhoria. Com o objetivo de aproximá-lo da perspectiva do cliente, o mapeamento é feito do fim do processo para o início (FORNO et al., 2014). De acordo com Bhamu e Sangwan (2014), a formação de uma equipe multidisciplinar contribui para que os objetivos da PE sejam atingidos. Sendo assim, à exemplo de Braglia (2006) e McDonald e Aken (2002), a equipe responsável pela execução do mapeamento é de natureza multidisciplinar, composta por pessoas de diferentes partes da organização. Por fim, nessa etapa o mapeamento é feito ainda sem considerar as incertezas presentes nos processos ou etapas.

$\mathrm{Na}$ etapa (iii) acontece o levantamento das principais fontes de incerteza relacionadas ao fluxo de valor mapeado, através de entrevistas semi-estruturadas com a liderança. Segundo Branski et al. (2015), entrevistas desse tipo possuem um conjunto de perguntas previamente definidas de forma a direcionar a discussão e ampliar a coleta de informações. No entanto, há liberdade para que o entrevistador conduza a conversa, permitindo uma maior riqueza nas informações obtidas. As incertezas elencadas devem ser apresentadas nessas entrevistas e classificadas em uma escala que varia de 0 a 10 , sendo 0 insignificante e 10 uma incerteza absolutamente crítica, de acordo com a experiência do entrevistado acerca do processo em questão. Os dados acerca do comportamento histórico das incertezas escolhidas são obtidos diretamente com a empresa em estudo. As fontes de incerteza cuja soma acumulada dos valores médios de criticidade, obtidos a partir das entrevistas semi-estruturadas com lideranças da empresa, totalizarem $80 \%$ (incertezas tipo "A") terão sua variabilidade estudada e inserida na análise do mapa atual.

$\mathrm{Na}$ etapa (iv) é feita a análise crítica do estado atual, utilizando como suporte a ferramenta de simulação de Monte Carlo (SMC). Segundo Kentel e Aral (2005), a SMC é utilizada como forma de quantificar a variabilidade presente em cenários probabilísticos através do uso de simulação computacional. A SMC tem sido utilizada 
em diferentes cenários que envolvam análise de riscos, tais como na saúde e na gestão de projetos (MIORANDO, 2010; OLDONI, 2017) permitindo o estudo das variabilidades que possam afetar os prazos finais. Nesse sentido, a SMC permite meios para estudar como a variabilidade das fontes de incerteza elencadas na etapa (iii) influenciam o lead time.

A etapa $(v)$ consiste no mapeamento do estado futuro almejado para o fluxo de valor. Este mapa futuro visa remover os desperdícios encontrados com foco tanto nos valores nominais dos processos quanto nas variabilidades das fontes de incerteza identificadas (BRAGLIA et al., 2009). A simbologia utilizada no mapa futuro é semelhante à utilizada no mapa atual, com a inserção de alguns ícones evidenciando as melhorias propostas. Rother e Shook (1999) propõem um conjunto de questões acerca do estado futuro de forma a auxiliar na sua construção. São elas: (a) qual o takt?; (b) os produtos acabados serão estocados ou serão imediatamente enviados?; (c) onde pode ser aplicado o fluxo contínuo de produção?; (d) existe a necessidade de um sistema puxado tipo supermercado no fluxo de valor?; (e) qual será o ponto na cadeia de produção que será utilizado como base para o planejamento da produção?; (f) como será feito o nivelamento do mix de produção no ponto que dita o ritmo do processo?; $(g)$ qual a unidade mínima de produção?; e $(h)$ qual o tipo de melhoria no processo será necessária?. Além disso, as fontes de incerteza que não forem abordadas nesta primeira instância de melhoria têm seus níveis de variabilidade mantidos igualmente no estado futuro. Contudo, ao consolidar o novo lead time objetivado, novamente todas as incertezas elencadas são consideradas através da SMC, análogo ao que foi realizado na etapa (iv).

Por fim, na etapa ( $v i)$ elabora-se um plano de ação de forma a alcançar as melhorias almejadas no mapa de estado futuro. Este plano deve ter suas metas, atividades e responsáveis claramente definidos, assim como os prazos a serem cumpridos. De forma a garantir o sucesso da implementação, reuniões de follow-up devem ser feitas entre a equipe responsável pela execução e a de mapeamento. Devem também ser listados os recursos necessários para a implementação do mapa futuro e elaborada uma apresentação para a diretoria. 


\section{RESULTADOS}

O método proposto foi aplicado em um hospital público universitário, localizado no município de Florianópolis, em Santa Catarina, que está em operação há 37 anos. A primeira etapa do método previa a escolha da família de produtos a ser tratada. Em reunião com a gerência do hospital, foi definido que o setor escolhido para o desenvolvimento do trabalho seria o de gerenciamento dos suprimentos do tipo órteses, próteses e materiais especiais (OPME), por ser um setor que lida com produtos de alto valor agregado e que carece de políticas de gestão de estoque. Os materiais são obtidos através de um pregão com validade de um ano e armazenados em um regime de consignação. O setor possui capacidade de armazenagem de aproximadamente 1500 unidades e atualmente trabalha com aproximadamente 300 itens distintos. O prazo de entrega acordado de forma contratual é de cinco dias. Os fornecedores possuem contratualmente a obrigação de trocar os itens consignados que estejam foram do prazo de validade, sem que exista um ônus direto para o hospital. Sendo assim, desenha-se um contexto onde não há incentivos para a gestão adequada dos produtos, com um grande potencial para oportunidades de melhoria na aplicação da metodologia aqui proposta. A demanda média dos itens analisados é de 215 unidades mensais, considerando o período de janeiro de 2016 a junho de 2017.

A segunda etapa correspondeu ao desenho do fluxo de valor do setor, feito através da abordagem determinística usual do MFV, apresentado na Figura 1. Para a elaboração do fluxo de valor foi formada uma equipe multidisciplinar, composta por funcionários do setor de OPME, assim como membros de todos os setores para quem o setor de OPME fornece produtos. Para gerar o mapa foram realizados três encontros de aproximadamente duas horas cada envolvendo os membros dessa equipe. Cabe destacar que os dados coletados para a elaboração do fluxo de valor são baseados em amostras disponíveis no momento do mapeamento e por consequência não refletem as variações que acontecem ao longo do tempo nesse fluxo.

Atualmente o setor não conta com uma política sistemática para o disparo de ordens de reposição com as empresas fornecedoras. Tal tarefa é feita com base na experiência do funcionário responsável. Quando o material é recebido, é feita a inspeção através de amostragem. Caso esteja de acordo, é então registrado no sistema e encaminhado ao estoque. Não existe uma forma sistemática de gerenciar as filas no estoque e são observadas ocorrências de produtos que atingem seu 
período de validade sem que sejam utilizados. Conforme comentando anteriormente, nessa situação a empresa fornecedora tem por obrigação contratual a troca do item sem ônus adicional para o hospital.

O setor de OPME atende diversas unidades do hospital, como por exemplo hemodinâmica e centro cirúrgico. As requisições feitas por cada uma dessas unidades não seguem uma forma padronizada, sendo feitas de diversas formas, como email, requisição física ou ligação telefônica. Uma vez recebidos esses pedidos, os itens são separados para aguardar a retirada em uma área específica de retiradas dentro do setor de OPME. Além do estoque dentro do setor de OPME, também existem estoques em algumas das unidades que são clientes do setor de OPME. Ao longo do fluxo de valor existem três pontos de programação: na solicitação, no recebimento e na retirada. Os itens são então retirados e após serem utilizados, é necessário laudo médico para atestar o uso e garantir a rastreabilidade do material. O lead time observado no processo é de 151 dias.

Uma vez que o mapa do estado atual foi desenhado com dados coletados de forma pontual, na terceira etapa foram levantadas as variabilidades presentes. Como esse processo é fundamentalmente voltado à gestão de materiais e fluxo administrativo, incertezas como máquina, manutenção e questões macroeconômicas puderam ser desconsideradas. Nesse sentido, através de entrevistas com os responsáveis, as incertezas tiveram seus níveis de criticidade classificados em uma escala de 0 a 10. Na Tabela 1 é apresentado um resumo dos resultados obtidos com as entrevistas. 
Figura 1 - Mapeamento do estado atual

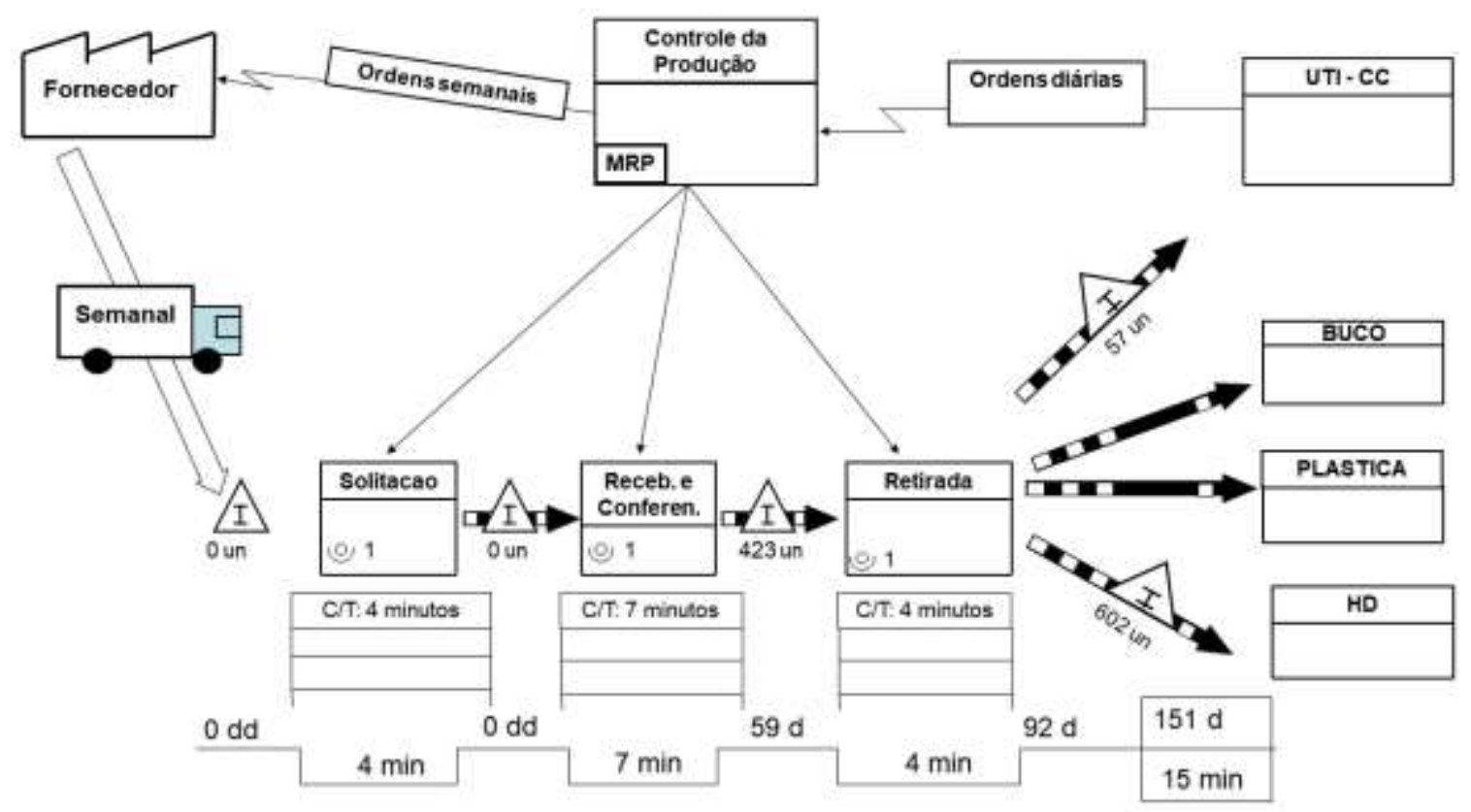

Tabela 1 - Níveis de incerteza

\begin{tabular}{clc}
\hline Grupo & \multicolumn{1}{c}{ Incertezas } & Nível de criticidade \\
\hline & Tempo de máquina & 0 \\
Incertezas da & Manutenção & 0 \\
organização & Prodidade & 1 \\
& Absenteísmo & 3 \\
& Acidentes & 1 \\
Incertezas da & Variação da demanda & 1 \\
cadeia de & Continuidade do fornecimento & 7 \\
suprimentos & Variação dos custos & 5 \\
& Cumprimento dos prazos pelos fornecedores & 0 \\
Incertezas & Políticas governamentais & 2 \\
externas & Questões macroeconômicas & 0 \\
& Desastres provocados pelo homem & 0 \\
& Desastres naturais & 0 \\
\hline
\end{tabular}

Dentre essas incertezas, as incertezas de variação da demanda, continuidade do fornecimento, produtividade dos trabalhadores e cumprimento dos prazos pelos fornecedores compõem as incertezas do tipo "A", sendo essas então as incertezas a serem incorporadas na análise. A coleta dos dados relativos a essas incertezas ocorreu em um período de trinta dias, onde foram realizadas tomadas de tempo de processo e contagem de estoque. Nos processos de solicitação, recebimento e retirada foram feitas medidas de tempo com o propósito de estudar as variabilidades que ocorrem na produtividade dos trabalhadores. Já para as demais incertezas 
classificadas como tipo "A", foram coletados valores acerca dos estoques existentes, tanto no setor de OPME quanto nas unidades cliente. Com base nesses dados coletados durante trinta dias, foram gerados histogramas e identificadas as distribuições de probabilidade de cada processo e estoques, apresentados na Tabela 2. A determinação das distribuições se deu através do uso do software ®Easyfit.

$\mathrm{Na}$ quarta etapa foi realizada a análise crítica do estado atual utilizando como suporte a SMC. Questões como a falta de sistemática para o pedido de reposição de itens com o fornecedor, a falta de sistemática para gerenciamento de filas no estoque e a falta de padronização na requisição de materiais por parte das unidades cliente podem ser constatadas através da abordagem determinística de análise do fluxo de valor.

Além dessas questões, é possível identificar outros pontos ao analisar o fluxo de valor sob um ponto de vista estocástico. Na Tabela 2 é possível observar que a etapa que mais agrega tempo ao lead time é o estoque nos clientes, contudo, essa etapa não é a que possui maior variabilidade. Ao considerar valores absolutos, o desvio padrão que apresentou maior valor foi o de estoque de MP, que representa a variabilidade presente na entrega do fornecedor. Essa variabilidade implica que o fornecedor possui uma frequência de entrega e/ou quantidade de produtos por entrega que apresentam grande oscilação. Sendo assim, é necessário identificar ações de melhorias junto ao fornecedor, de forma a diminuir essa variabilidade. Logo, ao analisar do ponto de vista estocástico, é possível observar que o maior estoque atual (clientes) possui uma baixa variabilidade e pode ser reduzido de forma agressiva. Tal ponto certamente não seria visto em um mapeamento feito de forma usual. Considerando a proporcionalidade de variação, o processo de recebimento é aquele que possui maior amplitude, o que pode gerar variações no lead time e dificuldades no balanceamento da mão de obra. Essa variabilidade pode acontecer por uma série de motivos, não só pela forma como os pedidos são realizados, mas também pelo nível de experiência do operador, a quantidade entregue pelo fornecedor em cada pedido e a falta de padrões no recebimento. Novamente, tal ponto não seria observado em um mapeamento determinístico.

Os dados presentes na Tabela 2 podem ser utilizados para validar percepções existentes entre os funcionários do setor de OPME. Uma das questões levantadas pela equipe do setor foi a falta de planejamento por parte das unidades que são 
abastecidas pelo setor de OPME. Contudo, essa falta de planejamento não é refletida na variação de demanda, tanto que o coeficiente de variação do estoque dos clientes é o mais baixo dentre os observados.

Identificados os parâmetros de cada uma dessas distribuições, a SMC foi então aplicada. Para cada distribuição foram gerados cinco mil dados aleatórios baseados nas funções de densidade de probabilidade de cada um dos processos. Somando-se os valores aleatórios gerados a cada ocorrência, tem-se cinco mil valores obtidos para o lead time total do processo. Esses cinco mil valores resultantes compõem a curva de probabilidade do lead time do processo, que juntamente ao histograma é apresentado na Figura 2. Na Tabela 3 são mostrados alguns pontos específicos de relevância, onde é possível observar a probabilidade de atendimento ao lead time. Com base nos valores obtidos através da SMC para o lead time do processo, é possível verificar qual a probabilidade de um certo valor de lead time ser atendido. Esse valor é dado pela função de densidade acumulada, mostrada na Figura 3.

Os valores apresentados na Tabela 3 evidenciam a importância da abordagem estocástica ao analisar o fluxo de valor. Cabe destacar que o valor encontrado com a metodologia usual de aplicação do MFV encontra-se abaixo do valor mínimo encontrado de acordo com a distribuição probabilística, o que corrobora para a afirmação de que a abordagem determinística representa uma condição irreal do fluxo de valor, a qual possui pouquíssimas chances de se repetir. Tal fato salienta a necessidade de incorporar estudos estocásticos na análise de fluxo de valor. Outro ponto de destaque é que para uma probabilidade de atendimento de $99 \%$, o lead time do processo é $44 \%$ maior que o previsto na abordagem determinística. Também é possível observar que um aumento de aproximadamente $7 \%$ no tempo de lead time eleva a probabilidade de atendimento de $70 \%$ para $99 \%$. Esses dois últimos pontos mostram que embora seja possível em metade dos casos obter lead times de até 196 dias, estabelecer um lead time de 217 dias permite que o prazo seja estimado de forma mais confiável, tornando o processo mais previsível.

$\mathrm{Na}$ quinta etapa a mesma equipe foi envolvida com o objetivo de elaborar um mapa de estado futuro para o fluxo de valor, identificando as oportunidades de melhoria existentes. foi elaborado o mapa de estado futuro apresentado na Figura 4. Por meio da abordagem determinística, foram identificadas algumas oportunidades de melhoria, tais como: elaboração de uma sistemática para definir pontos de pedido, de 
forma a melhorar o gerenciamento dos estoques; elaboração de uma sistemática para o gerenciamento das filas presentes no estoque, de modo que produtos com maior prazo de validade não sejam utilizados em detrimento daqueles com prazo menor; padronização das requisições de material recebidas pelo setor de OPME; e definir uma área de armazenamento de materiais de retirada específica para cada cliente.

De forma complementar, a abordagem estocástica permitiu identificar melhorias adicionais, tais como: padronização do processo de recebimento, de forma a aumentar sua previsibilidade e permitir um melhor balanceamento da carga de trabalho; redução dos níveis de estoque nas unidades cliente, devido à baixa variabilidade encontrada; e revisão junto aos fornecedores de sua política de entrega, de forma a diminuir a variabilidade. Por último, foi elaborado um plano de ação definindo prazos e responsáveis pela implementação das melhorias definidas. $\mathrm{Na}$ Figura 4 é possível observar as melhorias relacionadas à análise estocástica em amarelo e à análise determinística em azul.

Tabela 2 - Distribuições de probabilidade dos tempos de processos e estoques do fluxo de valor

\begin{tabular}{|c|c|c|c|c|c|}
\hline & Solicitação & Recebimento & Retirada & $\begin{array}{c}\text { Estoque } \\
\text { MP }\end{array}$ & $\begin{array}{l}\text { Estoque } \\
\text { Clientes }\end{array}$ \\
\hline Tipo & Johnson SB & $\begin{array}{c}\text { Generalized } \\
\text { Pareto }\end{array}$ & $\begin{array}{c}\text { Generalized } \\
\text { Extreme Value }\end{array}$ & $\begin{array}{c}\text { Johnson } \\
\text { SB }\end{array}$ & Burr \\
\hline Parâmetros & $\begin{array}{c}\gamma=0,907 \\
\delta=1,44 \\
\lambda=0,133 \\
\varepsilon=0,0323\end{array}$ & $\begin{array}{c}K=-0,382 \\
\sigma=0,142 \\
\mu=0,00671\end{array}$ & $\begin{array}{l}K=-0,348 \\
\sigma=0,0191 \\
\mu=0,0530\end{array}$ & $\begin{array}{c}\gamma=0,436 \\
\delta=0,759 \\
\lambda=798,27\end{array}$ & $\begin{array}{c}k=7,419 \\
\alpha=37,806 \\
\beta=3393,7 \\
\gamma=0\end{array}$ \\
\hline Média & 0,0801 & 0,1075 & 0,0589 & 1543,03 & 3177,93 \\
\hline $\begin{array}{l}\text { Desvio } \\
\text { Padrão }\end{array}$ & 0,0190 & 0,0770 & 0,0184 & 193,95 & 110,45 \\
\hline $\begin{array}{l}\text { Coeficiente } \\
\text { de variação }\end{array}$ & $23,7 \%$ & $71,6 \%$ & $31,3 \%$ & $12,6 \%$ & $3,5 \%$ \\
\hline
\end{tabular}


Figura 2 - Função de densidade de probabilidade do lead time

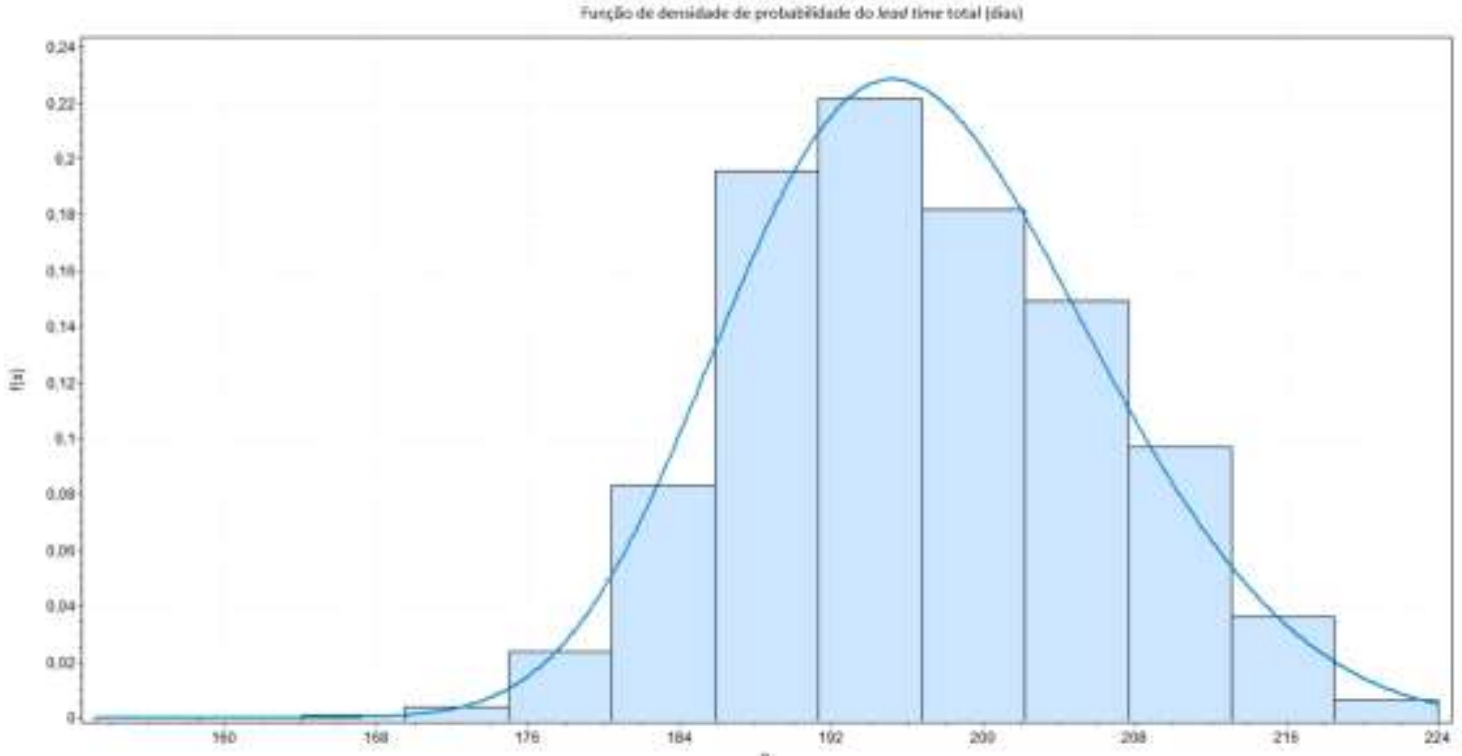

Figura 3 - Função de densidade acumulada do lead time

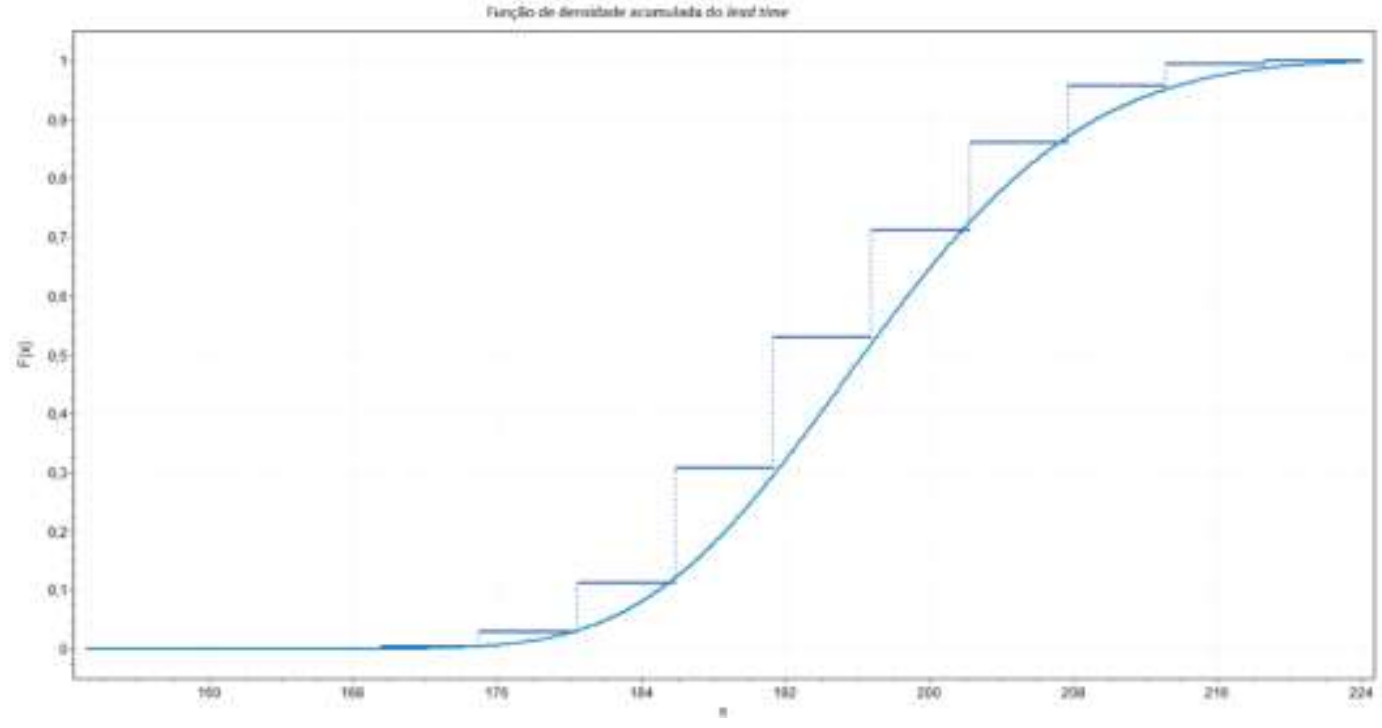

Tabela 3 - Probabilidade de atendimento ao lead time

\begin{tabular}{cccc} 
Cenários & Lead Time & Probabilidade de atendimento & Diferença \\
\hline Determinístico & 151 & - & - \\
1 & 196 & $50 \%$ & $30 \%$ \\
2 & 202 & $70 \%$ & $34 \%$ \\
3 & 210 & $90 \%$ & $39 \%$ \\
4 & 213 & $95 \%$ & $41 \%$ \\
5 & 217 & $99 \%$ & $44 \%$ \\
\hline
\end{tabular}


Figura 4 - Mapa do estado futuro

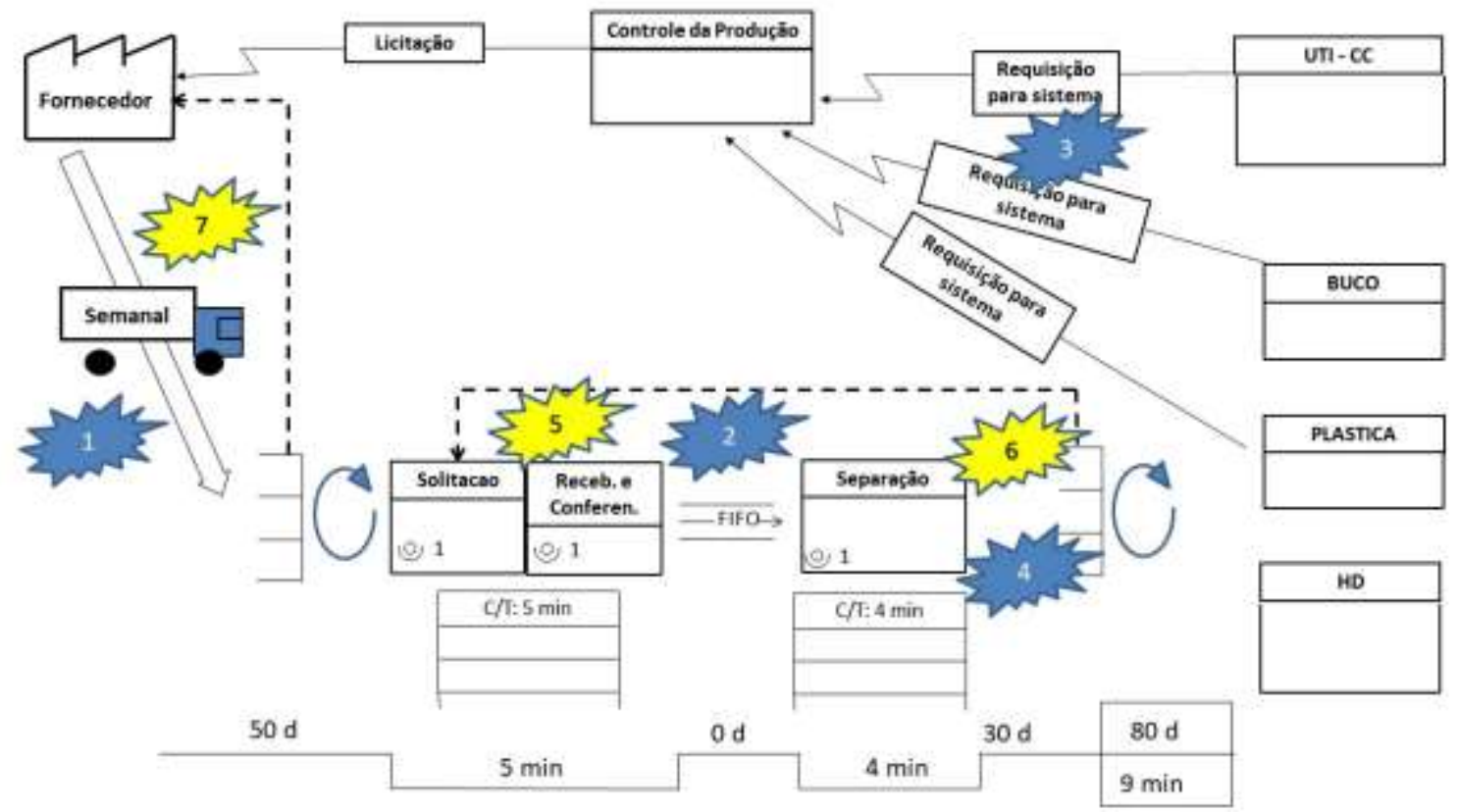

\section{CONCLUSÃO}

A elaboração desse trabalho permitiu o estudo do mapeamento de fluxo de valor sob uma perspectiva estocástica, considerando os aspectos dinâmicos que não são considerados quando o mapeamento é executado tradicionalmente. $O$ objetivo geral de propor um método de identificação de oportunidades de melhorias que combine o mapeamento de fluxo de valor e simulação de Monte Carlo foi atingido. Já em relação aos objetivos específicos, todos eles foram atingidos. Através de uma aplicação prática do método as fontes de incerteza no fluxo de valor foram identificadas e as distribuições de probabilidade dessas incertezas foram definidas.

Do ponto de vista teórico foi realizado um estudo acerca da PE, do MFV e incertezas. O levantamento bibliográfico sobre a PE permitiu a compreensão dos seus mais importantes princípios, aspectos e objetivos. Assim como foi possível levantar suas práticas mais utilizadas, dentre as quais destaca-se o MFV, que serve como base para a aplicação das demais práticas. No estudo sobre MFV foram identificadas as vantagens presentes em sua aplicação, do mesmo modo que as dificuldades enfrentadas e as limitações. Ainda na revisão bibliográfica foram apresentadas as fontes de incerteza presentes em sistemas de produção, nos diferentes níveis da cadeia de suprimentos. Através do estudo foram identificadas lacunas na aplicação do MFV, para as quais propõem-se a solução através da metodologia proposta, 
combinando a abordagem tradicional do MFV à simulação de Monte Carlo. Em relação às contribuições práticas, por meio da aplicação em um hospital universitário da metodologia proposta foi possível verificar que a aplicação dessa metodologia permite identificar características do processo que não poderiam ser identificadas em uma abordagem tradicional e definir ações para essas oportunidades de melhorias identificadas.

Dentre as limitações deste estudo cabe destacar a metodologia utilizada para a qualificação das incertezas presentes nos processos. A utilização da percepção dos gestores pode estar restrita ao nível de compreensão destes quanto ao fluxo de valor, implicado na potencial existência de incertezas que apresentam um caráter atípico, como incertezas relacionadas a decisões acíclicas. O método proposto para a consideração das incertezas também configura uma limitação, dado que apenas Monte Carlo foi utilizado para considerar as incertezas e variabilidades do fluxo de valor. Assim, outros métodos que poderiam ter resultados similares ou complementares não foram abordados neste trabalho. Além disso, em relação à coleta de dados, foi utilizado um histórico de trinta dias, o que faz com que a análise esteja restrita a esses dados. Não foram avaliados anos anteriores e nem consideradas previsões futuras.

Como sugestão de pesquisas futuras, sugere-se a aplicação da metodologia proposta em diferentes cenários, de forma a estender os limites do método além do caso prático mostrado nesse trabalho. Sugere-se também o desenvolvimento de uma metodologia para que a classificação da criticidade das incertezas seja feita de forma quantitativa e não apenas baseado na experiência dos gestores dos processos estudados. Por último, sugere-se a utilização de outras formas complementares à SMC para considerar as incertezas presentes no fluxo de valor..

\section{REFERÊNCIAS}

ABDULMALEK, F. A.; RAJGOPAL, J. Analyzing the benefits of lean manufacturing and value stream mapping via simulation: A process sector case study. International Journal of

Production Economics, v. 107, n. 1, p. 223-236, 2007.

https://doi.org/10.1016/i.ijpe.2006.09.009

BHAMU, J.; SANGWAN, K. S. Lean manufacturing: literature review and research issues. International Journal of Operations and Production Management, v. 34, n. 7, p. 876940, 2014. https://doi.org/10.1108/IJOPM-08-2012-0315 
BRAGLIA, M.; CARMIGNANI, G.; ZAMMORI, F. A new value stream mapping approach for complex production systems. International Journal of Production Research ISSN:, v. 44, n. 18-19, p. 3929-3952, 2006. https://doi.org/10.1080/00207540600690545

BRAGLIA, M.; FROSOLINI, M.; ZAMMORI, F. Uncertainty in value stream mapping analysis. International Journal of Logistics: Research and Applications, v. 126, n. 6, p. 435-453, 2009. https://doi.org/10.1080/13675560802601559

BRANSKI, R. M.; FRANCO, R. A. C.; JR., O. F. L. Metodologia de Estudos de Caso Aplicada à Logística. p. 1-12, 2015.

DETTY, R. B.; YINGLING, J. C. Quantifying benefits of conversion to lean manufacturing with discrete event simulation: A case study. International Journal of Production Research, v. 38, n. 2, p. 429-445, 2000. https://doi.org/10.1080/002075400189509

DICKSON, E. W. et al. Application of Lean Manufacturing Techniques in the Emergency Department. Journal of Emergency Medicine, v. 37, n. 2, p. 177-182, 2009. https://doi.org/10.1016/i.jemermed.2007.11.108

DUGGAN, K. J. Creating Mixed Model Value Streams: Practical Lean Techniques for Building to Demand. Productivity Press, 2002.

FLYNN, B. B.; SAKAKIBARA, S.; SCHROEDER, R. G. Relationship between JIT and TQM: Practices and Performance. Academy of Management Journal, v. 38, n. 5, p. 1325-1360, 1995.

FORNO, A. J. D. et al. Value stream mapping: A study about the problems and challenges found in the literature from the past 15 years about application of Lean tools. International Journal of Advanced Manufacturing Technology, v. 72, n. 5-8, p. 779-790, 2014. https://doi.org/10.1007/s00170-014-5712-z

GREEF, A. C.; FREITAS, M. DO C. D.; ROMANEL, F. B. Lean office: operação, gerenciamento e tecnologias. Atlas, São Paulo, SP, 2012.

HINES, P. et al. Value Stream Management. The International Journal of Logistics Management, v. 9, n. 1, p. 25-42, 1998. https://doi.org/10.1108/09574099810805726

HINES, P.; RICH, N. The Seven Value Stream Mapping Tools. International Journal of Operations \& Production Management, v. 17, n. 1, p. 46-64, 1997. https://doi.org/10.1108/01443579710157989

JIMMERSON, C.; WEBER, D.; SOBEK, D. K. Reducing waste and errors: piloting lean principles at Intermountain Healthcare. Journal on Quality and Patient Safety, v. 31, n. 5, p. 249-257, 2005. https://doi.org/10.1016/S1553-7250(05)31032-4

KENTEL, E.; ARAL, M. M. 2D Monte Carlo versus 2D Fuzzy Monte Carlo health risk assessment. Stochastic Environmental Research and Risk Assessment, v. 19, n. 1, p. 86-96, 2005. https://doi.org/10.1007/s00477-004-0209-1

LIAN, Y.-H.; VAN LANDEGHEM, H. Analysing the effects of Lean manufacturing using a value stream mapping-based simulation generator. International Journal of Production Research, v. 45, n. 13, p. 3037-3058, 2007. https://doi.org/10.1080/00207540600791590

MAPES, J.; SZWEJCZEWSKI, M.; NEW, C. Process variability and its effect on plant 
performance. International Journal of Operations \& Production Management, v. 20, n. 7, p. 792-808, 2000. https://doi.org/10.1108/01443570010330775

MARODIN, G. A.; SAURIN, T. A. Implementing lean production systems: research areas and opportunities for future studies. International Journal of Production Research, v. 51, n. 22, p. 6663-6680, 2013. https://doi.org/10.1080/00207543.2013.826831

MCDONALD, T.; AKEN, E. M. VAN. International Journal of Applications: A Leading Journal of Supply Chain Utilising Simulation to Enhance Value Stream Mapping: A Manufacturing Case Application. International Journal of Logistics Research and Applications, v. 5, n. 2, p. 213-232, 2002. https://doi.org/10.1080/13675560210148696

MIORANDO, R. Modelo econômico-probabilístico de análise de risco em projetos de TI. 2010.

OLDONI, V. P. Modelo Econômico-Probabilístico para Seleção e Priorização de Projeto de Produção Enxuta. 2017.

ROTHER, M.; SHOOK, J. Learning to See: Value Stream Mapping to Add Value and Eliminate MUDA. Lean enterprise institute, 1999.

SETH, D.; GUPTA, V. Application of value stream mapping for lean operations and cycle time reduction : an Indian case study. Production Planning \& Control: The Management of Operations, n. October 2012, p. 37-41, 2012. https://doi.org/10.1108/17410380810869950

SETH, D.; SETH, N.; GOEL, D. Application of value stream mapping (VSM) for minimization of wastes in the processing side of supply chain of cottonseed oil industry in Indian context.

Journal of Manufacturing Technology Management, v. 19, n. 4, p. 529-550, 2008.

SINGH, B.; GARG, S. K.; SHARMA, S. K. Value stream mapping: Literature review and implications for Indian industry. International Journal of Advanced Manufacturing Technology, v. 53, n. 5-8, p. 799-809, 2011. https://doi.org/10.1007/s00170-010-2860-7

TEGNER, M. G. et al. Lean office e BPM: proposição e aplicação de método para a redução de desperdícios em áreas administrativas. Revista Produção Online, v. 16, n. 3, p. 10071032, 2016. https://doi.org/10.14488/1676-1901.v16i3.2308

TORTORELLA, G. L. et al. Making the value flow: Application of value stream mapping in a department of a Brazilian public healthcare organization. 23rd International Conference for Production Research, ICPR 2015, v. 3363, n. March, 2016.

TYAGI, S. et al. Value stream mapping to reduce the lead-time of a product development process. International Journal of Production Economics, v. 160, p. 202-212, 2015. https://doi.org/10.1016/.i.jpe.2014.11.002

WEE, H. M.; WU, S. Lean supply chain and its effect on product cost and quality: a case study on Ford Motor Company. Supply Chain Management: An International Journal, v. 14, n. 5, p. 335-341, 2009. https://doi.org/10.1108/13598540910980242

WOEHRLE, S. L.; ABOU-SHADY, L. Using Dynamic Value Stream Mapping And Lean Accounting Box Scores To Support Lean Implementation. American Journal of Business Education AJBE, v. 3, n. 8, p. 67-76, 2010. https://doi.org/10.19030/ajbe.v3i8.472

WOMACK, J. P.; JONES, D. T. A mentalidade enxuta nas empresa: Elimine o 
desperdício e crie riqueza. Elsevier, 2004.

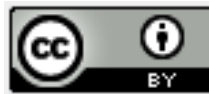

Artigo recebido em: 03/11/2017 e aceito para publicação em: 09/07/2018 DOI: http://dx.doi.org/10.14488/1676-1901.v18i3.3052

Revista Produção Online. Florianópolis, SC, v. 18, n. 3, p. 995-1015, 2018. 